\title{
On the Intermediary Function of Coping Styles: Between Self-Concept and Subjective Well Being of Adolescents of Han, Qiang and Yi Nationalities
}

\author{
Tianmei Zhou ${ }^{1 *}$, Dingchu $\mathrm{Wu}^{2}$, Lin $\operatorname{Lin}^{1}$ \\ ${ }^{1}$ Institute of the Educational Science, Neijiang Normal University, Neijiang, China \\ ${ }^{2}$ Institute of the Educational Science, Sichuan Normal University, Chengdu, China \\ Email: "zhtm6566@yahoo.cn
}

Received October $24^{\text {th }}, 2011$; revised November $23^{\text {rd }}, 2011$; accepted December $27^{\text {th }}, 2011$

\begin{abstract}
In order to know the influence of adolescents' self-concept on the subjective well being and the function of coping styles in them, the author adopted the Tennessee Self-Concept Scale, Simplified Coping Style Scale and the Questionnaire on the Subjective Euphoria, and made a survey on six middle schools of Sichuan province and got back 867 valid answered questionnaires. The results show that 1) differences are obvious in self-concept, coping styles and subject well being among adolescents of Han, Qiang and Yi nationalities; 2) the positive self-concept exerts a notable positive influence on subjective well being, and the negative exerts a negative one; 3 ) the coping styles play an intermediary role in the influence of self-concept on the subjective well being. On the above basis, the author concludes that the self-concept exerts influence on the subjective well being directly as well as indirectly through the mediation of coping styles.
\end{abstract}

Keywords: Adolescents; Self-Concept; Coping Style; Subjective Well Being

\section{Introduction}

As the core of personality, the self-concept is the knowledge about one's own character, ability, appearance, attitude, emotion and values. And it is the cognition on one own as the objective party. It is usually treated as a psychological variant in self evaluation. The subjective wellbeing is an important comprehensive psychological index for judging people's quality of life $(\mathrm{Wu}, 2000)$. This includes cognition and emotion. The former is aiming at the individual's satisfaction with life, which involves the general life satisfaction and the life satisfaction with specific aspect. And the latter refers to the individual's emotional experience which has two subtypes: positive emotions and negative emotions (Diener, 1984). T. Terry and Huebner (1995) showed in their study that subjective wellbeing and self-concept are the two different categories which, however, are in certain relation with each other, namely, the more positive the self-concept is, the stronger the subjective wellbeing will become. Some studies indicate that the various factors of self-concept are in positive correlation with all the other aspects except symmetrical emotion. As to the prediction for indexes A and $\mathrm{B}$ of degree of life satisfaction, the self-physiology and self-psychology in the self-concept have the striking predicting effect ( $\mathrm{Li}, 2006)$, i.e. the higher one's self cognition is, the higher the index of degree of his life satisfaction will become.

The coping strategy is the cognitive and behavioral means an individual takes when facing a stressful situation or event (Tong, 2004). As an important intermediary in the stress action, it plays a protective role either physical or mentally (Wang, Wang, \& Ma, 1999). The relevant studies show that the individual's self-concept is in a striking positive correlation with

${ }^{*}$ Corresponding author. coping strategies. For instance, the research reveals that the various dimensions of rural children's self-concept are in striking negative correlation with the negative coping strategy, but in positive correlation with the positive one (Wang \& Fan, 2009). The researches made on both university students and middle school ones also show that all the positive factors in the self-concept are in a striking correlation with the positive coping strategy and in a negative correlation with the negative coping one. Further, the self-critic factor is in a striking positive correlation with the negative coping strategy (Wang, 2001; Zheng, Hao, \& Hou et al., 2008; Dan, 2009). Meanwhile, the coping strategy is in a striking correlation with all the dimensions of degree of life satisfaction. The more frequently an individual employs coping strategies as problem solving and help seeking, the higher his or her well being will be. On the contrary, the more frequently an individual employs the coping strategies as escaping, bad temper and abreaction, the lower his or her well being will become. This shows that the coping strategy plays a role in prediction for the subjective wellbeing (Rim, 1993; Wang \& Ding, 2003; Yang \& Shi, 2004). Accordingly, the positive coping strategy is in a positive correlation with the subjective well being, and the negative coping one is in a negative correlation with the subjective well being. Fundamentally, the cultivation of positive coping strategy leads to the promotion of well being; however, the coping strategies as self-accusation and escaping hinder the promotion of well being (Ren \& Ye, 2006; Qiu, Zhang, \& Yao, 2007; Qiu, 2009).

To sum up, the past studies were made on the direct relations between self-concept, subjective well being and coping styles from the perspective of two parties. And they lack analysis of the role of mediation of the two. Do coping styles as a mediation variant play their role in the relation between the self- 
concept and subjective well being? For this consideration, the study has brought the adolescents' coping styles into the relation between self-concept and subjective well being and examines the self-concept's influence on the subjective well being. In addition, it probes into the shifting mechanism from selfconcept to the subjective well being. If the supposition that coping strategies play an intermediary role between the selfconcept and the subjective well being is proved, this may serve as a piece of evidence for the promotion of adolescents' well being and their health education.

The paper has the following hypotheses: cultural factors may exert influence on adolescents' self-concepts, coping styles and their subjective well being. Coping styles play the mediation role between the adolescents' self-concepts and subjective well being of Han, Qiang and Yi.

\section{Method}

\section{Participants}

Participants have been drawn after layering. One middle school has been chosen from Liangshan Prefecture in which Yi people assemble, Beichuan County, Mianyang, in which Qiang people mainly live, Ziyang City and Neijiang City respectively. And 18 classes have been chosen randomly from grade 7 to grade 12 in these schools. To them 900 questionnaires have been handed out, among which 895 have been taken back. Except those incomplete ones, 867 are valid, among which 442 are of Han nationality and 231 are of Qiang nationality, 193 are of Yi nationality; 352 are of boys and 515 are of girls; 300 are of city students and 565 are of rural students. There are 122, 148, 134, 139, 232 and 91 students investigated from grade 7 to grade 12 respectively. And their ages vary from 11 to 19 (15.6 $\pm 1.6)$.

\section{Research Materials}

Self-Concept Scale: Tennessee Self-Concept Scale was devised by American psychologist H. F. Wiliams and revised as the 3 rd edition by a Taiwanese Lin Bangjie, which contains 70 items (Lin, 1980). This scale includes ten aspects as two dimensions of self-concept and comprehensive ones, of which structural dimension involves identity, self satisfaction and self behavior, content dimension has physical self, moral self, psychological self, family self and social self, total includes self score and self criticism. The higher the first nine aspects score, the more positive the self-concept becomes, and the higher score self criticism gets, the more negative the self-concept will be. The relevant study (Fan, 2002) indicates Cronbach's is 0.87 and the Spearman-Brown's split half reliability is 0.97 . This study divides self-concept into two types: the positive one and the negative one. The former includes physical self, moral self, psychological self, family self and social self while the latter involves self criticism. The Cronbach's of the scale is 0.90 and the Spearman-Brown's split half reliability is 0.88 .

Coping Style Questionnaire: The simplified coping questionnaire (Wang, Wang, \& Ma, 1999) (SCSQ) has been employed for evaluation. The questionnaire is made up of two dimensions: positive coping style and negative coping style, 20 items altogether. The positive coping style ranges from items 1 to 12 , which mainly reflects its main features. And the negative coping style is from items 13 to 20 . They mainly show the features of negative coping style. Moreover, they have relatively high reliability and validity. The Cronbach's $\alpha$ of positive coping style and that of negative coping style are 0.75 and 0.76 respectively.

Subjective Well Being Scale: This study adopts China Multidimensional Life Satisfaction Scale for adolescent (CMSLSS) devised by Zhang, X. G. (Zhang, He, \& Zheng, 2004) after Huebner's Multidimensional Life Satisfaction Scale for Adolescent (Huebner, 1994) and Bradburn's Affect Scale (Wang, Wang, \& Ma, 1999). And CMSLSS employs 7-rank system of scoring, the lowest score of each item is 1 , the highest is 7 . The higher the score is, the higher the degree of life satisfaction will be. The Cronbach's $\alpha$ of this scale is 0.85 . Further, there are 10 yes-no questions in Affect Scale, of which 5 are for describing the positive emotion and the other 5 for describing the negative emotion. The answer "yes" for the positive emotion question is scored 1, and the answer "no" scored 0. And the answer "yes" for the negative emotion question is scored 0 , and the answer "no" scored 1.

These materials are confined to the people over 12 .

\section{Procedure and Data Analysis}

Using stratified cluster sampling method and anonymous test by class, we regained questionnaires at once after test. Before test, the examiners directed students how to rightly fill the forms under the instructions. After students had understood rules fully, the test began. The head teacher of each class acted as examiner and was trained in advance.

In our study, data was processed and analyzed by SPSS 13.0 and AMOS 7.0 for Windows.

\section{Results}

\section{A Comparison of Self-Concepts, Coping Styles and Subjective Well Being among Adolescents of Han, Qiang and Yi Nationalities}

The self-concept, coping style and subjective well being of adolescents are of sharp difference among nationalities. The SLD analysis on covariance reveals (Table 1) that in terms of self-concept the scores of positive self-concept from the high to low follow the order of Han, Yi and Qiang. In addition, the average score of positive self-concept of Han and Yi adolescents is strikingly higher than that of Qiang adolescents. And the average score of negative self-concept of $\mathrm{Yi}$ adolescents is noticeably lower than that of Han and Qiang adolescents. The average scores of life satisfaction and negative emotion are higher than those of Han and Qiang adolescents.

\section{A Relevant Analysis on Self-Concept, Coping Style and Subjective Well Being}

Table 2 shows that the adolescents' positive self-concept is in positive correlation with various dimensions of positive coping style and subjective well being, but in negative correlation with negative coping style. And their negative self-concept is in positive correlation with their negative coping styles, but in negative correlation with degree life satisfaction and negative emotion. The various dimensions of subjective well being are in positive correlation, but in negative correlation with negative coping style. 


\section{The Intermediary Function of Coping Style between Self-Concept and Subjective Well Being}

In the examination of the influence of the predictable variable on the dependent variable, if the predictable variable exerts influence on the dependent variable through variable $\mathrm{M}, \mathrm{M}$ will be called mediated variable. The relationships among three variables need to be studied in testing of the intermediary effect or function. Supposing that the predictable variable is in striking correlation with the dependent variable, and it is also in striking correlation with the mediated variable, if the correlation or regression coefficient between the predictable variable and dependent variable decreases when the mediated variable is added, people will believe that the intermediary function is obvious. When the regression coefficient lowers to 0 , this will be called complete intermediary function (Xin, Guo, \& Chi, 2007). The statistics in Table 2 show that the intermediary function can be tested. Meanwhile, we have constructed the model without mediation as a competitive one according to some relevant theories. And through the comparison between fit indexes, we made attempts to find which model fits better with the real data. The difference between the model without mediation and the one with it lies in whether the influence of the ado- lescents' self-concepts on their subjective well being is through the mediation of the coping styles.

The Table 3 shows that the various indexes of the model with mediation exceed the critical value and are better than their counterparts of the one with the mediation. This shows that the fitting effect of the model with mediation is good, that is, the coping styles play the mediation role between the adolescents' self-concepts and their well being.

In order to know the role the coping style plays in the relation between self-concept and subjective well being, the author first centralizes various dimensions of self-concept, coping style and subjective well being and then gives them a regression analysis. In the first step, he has examined predictable role both positive and negative self-concepts play on various dimensions of subjective well being. In the second step, he has tested the predictable function self-concept has exerted on coping style as mediated variable (the predictable functions of positive and negative self-concept on positive coping styles are respectively ( $\beta=0.47, P<0.001 ; \beta=0.10, P<0.05)$. The predictable functions of positive and negative self-concept on negative coping styles are respectively $(\beta=-0.24, \beta=0.39, P<0.001)$. In the third step, the author takes degree of life satisfaction of subject-

Table 1.

Scores of self-concepts, coping styles and subjective well being among adolescents of Han, Qiang and Yi nationalities.

\begin{tabular}{|c|c|c|c|c|c|c|c|}
\hline \multirow[t]{2}{*}{ Scale } & \multirow{2}{*}{$\begin{array}{c}\text { Han (1) } \\
(\mathrm{N}=442)\end{array}$} & \multirow{2}{*}{$\frac{\mathrm{Yi}(2)}{(\mathrm{N}=231)}$} & \multirow{2}{*}{$\begin{array}{l}\text { Qiang (3) } \\
(\mathrm{N}=193)\end{array}$} & \multirow[t]{2}{*}{$\mathrm{F}$} & \multicolumn{3}{|c|}{ Pair Comparison $(P<0.05)$} \\
\hline & & & & & (1)(2) & (2)(3) & (1)(3) \\
\hline Pos Self Con & $3.68 \pm 0.39$ & $3.62 \pm 0.36$ & $3.52 \pm 0.38$ & $9.62^{* * *}$ & & (2) $>$ (3) & (1) $>$ (3) \\
\hline Neg Self Con & $3.24 \pm 0.63$ & $2.85 \pm 0.64$ & $3.17 \pm 0.58$ & $24.36^{* * *}$ & (1) $>$ (2) & (3) $>$ (2) & \\
\hline Pos Coping Style & $1.89 \pm 0.44$ & $1.93 \pm 0.42$ & $1.86 \pm 0.43$ & 1.19 & & & \\
\hline Neg Coping Style & $1.35 \pm 0.57$ & $1.28 \pm 0.49$ & $1.50 \pm 0.53$ & $8.84^{* * *}$ & & (3) $>$ (2) & (3) $>$ (1) \\
\hline Life Sat & $4.50 \pm 0.69$ & $4.77 \pm 1.73$ & $4.49 \pm 0.61$ & $5.30^{* *}$ & (2) $>$ (1) & (2) $>$ (3) & \\
\hline Pos Emo & $2.86 \pm 0.97$ & $2.74 \pm 1.02$ & $2.81 \pm 0.99$ & 0.87 & & & \\
\hline Neg Emo & $3.02 \pm 1.43$ & $3.49 \pm 1.34$ & $3.04 \pm 1.44$ & $7.92^{* * *}$ & (2) $>$ (1) & (2) $>$ (3) & \\
\hline
\end{tabular}

Note: ${ }^{*} P<0.05,{ }^{* *} P<0.01,{ }^{* * *} P<0.001$, Pos $=$ positive, Con $=$ concept, Neg = negative, Sat $=$ satisfaction, Emo $=$ emotion.

Table 2.

Correlation among self-concept, coping style and subjective well being.

\begin{tabular}{|c|c|c|c|c|c|c|c|}
\hline & Pos Self Con & Neg Self Con & Pos Coping S. & Neg Coping S. & Life Sat & Pos Emo & Neg Emo \\
\hline Pos Self Con & 1 & & & & & & \\
\hline Neg Self Con & $-0.14^{* *}$ & 1 & & & & & \\
\hline Pos Coping S. & $0.42^{* *}$ & 0.01 & 1 & & & & \\
\hline Neg Coping S. & $-0.29^{* *}$ & $0.35^{* *}$ & 0.04 & 1 & & & \\
\hline Life Sat & $0.50^{* *}$ & $-0.21^{* *}$ & $0.34^{* *}$ & $-0.18^{* *}$ & 1 & & \\
\hline Pos Emo & $0.23^{* *}$ & $0.07^{*}$ & $0.17^{* *}$ & 0.04 & $0.23^{* *}$ & 1 & 1 \\
\hline Neg Emo & $0.34^{* *}$ & $-0.34^{* *}$ & $0.21^{* *}$ & $-0.24^{* *}$ & $0.36^{* *}$ & 0.13 & \\
\hline
\end{tabular}

Note: S. = Style.

Table 3.

Models of coping styles' mediation role and their fit indexes.

\begin{tabular}{ccccccc}
\hline Mode 1 & $\chi^{2}$ & df & NFI & IFI & CFI & RMSEA \\
\hline Model 1 (without mediation) & 33.25 & 3 & 0.891 & 0.900 & 0.895 \\
Model 2 (mediation) & 69.77 & 8 & 0.914 & 0.906 & 0.904 & 0.090 \\
\hline
\end{tabular}


tive well being and positive and negative emotion as dependent variable and puts self-concept and coping style into equation.

Figure 1 and Table 4 and Table 5 can show that positive self-concept exerts a positive influence on subjective well being and negative self-concept has a negative influence on subjective well being (except positive emotion). Furthermore, both positive and negative coping styles partly play an intermediary role between self-concept and subjective well being. Thus the hypothesis has been brought to truth. The proportions of inter-mediary function of positive coping style between positive self-concept and degree of life satisfaction, positive emotion and negative emotion, and between negative self-concept and positive emotion are respectively $3.8 \%, 29 \%, 20 \%, 9.1 \%$; the proportions of intermediary function of negative coping style between negative self-concept and positive emotion, positive self-concept and negative emotion are respectively $18 \%$ and $7 \%$.

\section{Discussion}

\section{Nationalities' Influence on Adolescents' Self-Concept, Coping Style and Subjective Well Being}

All statistics of this study show that the ethnic cultural factors exert great influence on adolescents' self-concepts, coping styles and subjective well being. And scores of various dimen- sions of adolescents' self-concepts, coping styles and subjective well being are different with different nationalities. In addition, such differences are quite striking. This conclusion supports the hypothesis that there are differences of self concepts $(\mathrm{Hu}, 2000$; Mu, 2000; Zhou, Gao, \& Liu, 2005), coping styles (Zhou, 2009) and subjective well being (Ren \& Ye, 2006; Zhu, Miao, \& Chen, 2008; Gu \& Luo, 2009) between cultures. This may be brought about by the combination of different cultures, social standards, values, social economic status, family cultivation styles, peers' attitudes and schooling styles of different nationalities. This combination has become the social background of self evaluation of self-concept, coping style and subjective well being.

And China is a country with many nationalities. When all the other nationalities are under the influence of the Han culture, they still keep their own unique cultures which bring deep influence not only on their external behaviors and life styles but on changes of their knowledge and skill, behavioral tendency, self-concepts and both physical and mental health.

The differences of self-concepts among Han, Qiang and Yi people in Sichuan, China may be related to the following factors. Firstly, these people live in different environments and they have different religious beliefs and languages. Both Qiang and Yi are the two old nationalities on plateau. They usually live in the rugged environment like high mountains and river valley. This leads to their low productive force for a very long



Figure 1.

Model of part of mediated function of coping style between self-concept and subjective well being. Notes: 1) All numerical values in the model are standardized path coefficient. 2) The absolute value of $t$ of each path is between $2.96-12.86$. 
Table 4.

Intermediary function of positive coping style.

\begin{tabular}{cccc}
\hline Dep Var & $\begin{array}{c}\text { Co of Pre Func of Pos (m1) and } \\
\text { Neg Self }(\mathrm{m} 2) \text { without Coping S. } \beta\end{array}$ & $\begin{array}{c}\text { Co of Pre Func of } \\
\text { Pos Coping S. } \beta\end{array}$ & $\begin{array}{c}\text { Co of Pre Func of Pos (m1) and } \\
\text { Neg Self (m2) with Pos Coping S. } \beta\end{array}$ \\
\hline Life Sat & $\mathrm{m} 1=0.26^{* * *}, \mathrm{~m} 2=-0.15^{* * *}$ & $0.19^{* * *}$ & $\mathrm{~m} 1=0.25^{* * *}, \mathrm{~m} 2=-0.15^{* * *}$ \\
Pos Emo & $\mathrm{m} 1=0.24^{* * *}, \mathrm{~m} 2=0.11^{* *}$ & $0.16^{* * *}$ & $\mathrm{~m} 1=0.17^{* * *}, \mathrm{~m} 2=0.10^{* * *}$ \\
Neg Emo & $\mathrm{m} 1=0.30^{* * *}, \mathrm{~m} 2=-0.35^{* * *}$ & $0.21^{* * *}$ & $\mathrm{~m} 1=0.24^{* *}, \mathrm{~m} 2=-0.37^{* * *}$ \\
\hline
\end{tabular}

Note: Dep $=$ dependent, Var $=$ variable, $\mathrm{Co}=$ coefficient, Pre $=$ predictable, Func $=$ function.

Table 5.

Intermediary function of negative coping style.

\begin{tabular}{cccc}
\hline Dep Var & $\begin{array}{c}\text { Co of Pre Func of Pos (m1) and } \\
\text { Neg Self (m2) without Coping S. } \beta\end{array}$ & $\begin{array}{c}\text { Co of Pre Func of } \\
\text { Neg Coping S. } \beta\end{array}$ & $\begin{array}{c}\text { Co of Pre Func of Pos (m1) and } \\
\text { Neg Self (m2) with Neg Coping S. } \beta\end{array}$ \\
\hline Life Sat & $\mathrm{m} 1=0.26^{* * *}, \mathrm{~m} 2=-0.15^{* * *}$ & $-0.12^{* *}$ & $\mathrm{~m} 1=0.26^{* * *}, \mathrm{~m} 2=-0.15^{* * *}$ \\
Pos Emo & $\mathrm{m} 1=0.24^{* * *}, \mathrm{~m} 2=0.11^{* *}$ & 0.03 & $\mathrm{~m} 1=0.25^{* * *}, \mathrm{~m} 2=0.09^{*}$ \\
Neg Emo & $\mathrm{m} 1=0.30^{* * *}, \mathrm{~m} 2=-0.35^{* * *}$ & $-0.24^{*}$ & $\mathrm{~m} 1=0.28^{* * *}, \mathrm{~m} 2=-0.35^{* * *}$ \\
\hline
\end{tabular}

time. They believe in animism and natural things on which they think there is certain secret power that can determines people's fate. People are not allowed to have either words or behavior that may violate their will. The human's power is so weak before nature. These help to account for the fact that the scores of Qiang and Yi adolescents in self-concepts are unexceptionally lower than those of Han adolescents. On the other hand, both Qiang and Yi people are the old nationalities on plateau and they are characterized by farming and animal husbandry culture. Although Yi people worship Nature and their ancestors, in the long period of fighting with nature they have come to know more about it as well as causes of some natural phenomena. Accordingly, they no longer pin their hope on nature and gradually they are aware of their own powers (Qiu, 2006). For instance, the Yi family system is a particular social one which plays an important role in maintaining families' private properties, stabilizing whole families, establishing public morality, and choosing one's spouse (Yang, 2008). However, limited by the lever of productive force, their ability to know about nature is still rather weak. Comparatively, the Qiang people's belief is even more primitive and their conventions are characterized by the thick divine color. They worship gods of the heaven, the earth, the mountains, their villages and all the other gods in the nature. Thus, they have no fixed idol which is fused in the fete rites which mostly standardize people's behaviors, as forest protection, punishment of stealth, family and marriage harmonization, road and bridge building, and guesting principle (Zhao \& Luo, 2010). Both the two nationalities have their own languages. The Yi people speak the Nang language, but the Qiang people do not have the language specific to their own and they speak Chinese. WANG Mingke holds that to the most Qiang people the biggest dilemma for self identity is the language problem. The Qiang people call themselves "Erma" which is defined as the people who can speak the same language. However, the smaller dialects of different valleys are often not intelligible (Zhao \& Luo, 2010). "In the past, the Qiang history was written not in their own language but in other languages. The modern construction of Qiang culture and history somewhat improves such self identity, but such multi-culture tends to be exemplified and it even tends to disappear" (Zhao \& Luo, 2010). Therefore, what is mentioned above makes the Qiang adolescents lack sufficient knowledge of positive self-concept.

Secondly, the family economic pressure exerts great influence on adolescents' psychological development. The pressure the adolescents of minority nationalities (in rural areas in particular) face is much greater than that of Han adolescents. There are several reasons. The first reason is that the lag-behind of fundamental education of minority nationalities leads to the universal low level of these people's education, and hence leads to their low income and social status. The second lies in the fact that the privileged birth policy permits the people of minority nationalities to give two or three birth to children. The excessive economic pressure does not good to parents' health and their marriage, so parents may easily adopt some improper educational styles which are prone to children's problems of self development (Zhang \& Zhao, 2007). Comparatively, though the Han culture has long been around the Confucianism as its core values which highly stresses collectivism and social value, in the current society with the deepened reform and opening and the flood-in of Western thoughts, while maintaining the traditional collectivism oriented values, the Han adolescents have gradually begun to think of their self value. Accordingly, their positive evaluation of self-concept is the highest among the three nationalities.

\section{The Relationship between Self-Concept and Subjective Well Being of Han, Yi and Qiang and Partial Intermediary Role of Coping Style}

In this study, the subjective well being is in striking positive correlation with positive self-concept, but in striking negative correlation with negative self-concept. This is in accordance with conclusions of some relevant studies (Fredrickson, 1998; Li, 2006; Zhang \& Xu, 2007; Ling, Zhu, \& Liu, 2008; Wang, 2008; Wu, 2008). But then, how is the role of self-concept in subjective well being played? The statistic analysis shows that the variable coping style has partial intermediary role between self-concept and subjective well being, among which the intermediary role of positive coping style between self-concept and subjective well being reaches $29 \%$. The role of adolescents' self-concept in subjective well being is to promote or restrain subjective well being either through direct ways or indirect ways.

The Fredrickson's extended construction theory can be used 
to account for the function of self-concept on subjective well being and the role of coping style between self-concept and subjective well being. Fredrickson (1998) holds that various specific positive emotions, like joy, interest, satisfaction, pride and love, can extend people's instant cognitive ability, construct and promote people's personal resources (as to promote people's physical power and intelligence, psychological adjusting ability and social coordination), and improve people's subjective well being. Psychologists believe self-concept is the core of personality and it will exert influence on one's psychology and behavior as a core driving force. Further, it can provide help either psychological or environmental to strengthen people's self identity and hence enjoy themselves, promote their self esteem and confidence. The individual with a good self-concept can have relatively high positive emotion and low negative emotion (Li, Gan, \& Han, 2010). The high and much positive emotion is helpful for an individual to strengthen the social link with others. When needed, such social resources may become the source of social support and they can play a role in promoting one's subjective well being. And positive self-concept can extend one's horizon, stimulate one's creative thinking, thus the individual can better cope with pressure and confusion. Due to differences in depth of thoughts, flexibility and creativity of thinking, and social coping resources, individuals with different self-concepts may adopt different coping styles when facing pressure and difficulties. The adolescents with positive self-concept tend more to treat pressure as a challenge and they will seek for support both emotional and financial, positive causes and coping styles and plans. In this way, they keep high subjective well being. In contrast, the adolescents with negative self-concept usually undervalue and blame themselves. They think they are better than no one, so they take negative coping style, which makes them live in anxiety, low mood and helplessness. This will inevitably influences their subjective well being.

There is an interesting finding in this study. Positive selfconcept and positive coping style are in striking correlation with negative emotion, so is negative self-concept with positive emotion. The safe explanation for such a finding lies in the reason that individuals with positive self-concept are more confident. As a result, they have the courage to face challenge and apply themselves to the exploration of the unknown world which may bring success as well as failures. This in turn increases the chances for individuals to experience the negative emotion. On the contrary, for lack of confidence, individuals with negative self-concept may take escape or neglect mechanism in order to get psychological balance. This reduces individuals' chances of experiencing confusion, frustration and failures, which means the increase of chances for individuals to experience positive emotion.

On the whole, the choice of coping styles is an important way by which adolescents' self-concept influence their subjective well being. This finding further deepens the knowledge and understanding of relationship between adolescents' selfconcept and subjective well being. Meanwhile, it implies that people may improve and promote their subjective well being through the purposeful guidance and encouragement of individuals to use positive coping style efficiently.

\section{Conclusion}

There exist differences of adolescents' self-concepts, coping styles and subjective well being among nationalities. The average scores of positive self-concept of Han and Yi adolescents are noticeably higher than those of Qiang adolescents. The scores of negative self-concept of Yi adolescents are strikingly lower than those of Han and Qiang adolescents. The scores of negative coping style of Han and Yi adolescents are lower than those of Qiang adolescents. The degree of life satisfaction and negative emotion of $\mathrm{Yi}$ adolescents are higher than Han and Qiang adolescents.

Self-concept and coping style are in striking correlation with subjective well being. Positive self-concept is in negative correlation with subjective well being. And negative self-concept has obvious negative influence on subjective well being. Coping style plays a partial intermediary role in the influence of self-concept on subjective well being.

\section{REFERENCES}

Dan, W. J. (2009). A correlative research on undergraduate's selfconcept and confidence level and coping styles. Journal of Jiangsu Institute of Education (Social Science Edition), 25, 39-41.

Diener, E. (1984). Subjective well-being. Journal of Psychological Bulletin, 95, 542-575. doi:10.1037/0033-2909.95.3.542

Fan, F. M. (2002). The study on psychological health education of undergraduates. Beijing: Tsinghua University Press.

Fredrickson, B. L. (1998). What good are positive emotions? Review of General Psychology, 2, 300-319. doi:10.1037/1089-2680.2.3.300

Gu, Y. Y., \& Luo, Y. J. (2009). Neural mechanism of subjective well-being. Advances in Psychological Science, 17, 957-963.

Huebner, E. S. (1994). Preliminary development and validation of a multidimensional life satisfaction scale for children. Psychological Assessment, 6, 149-158. doi:10.1037/1040-3590.6.2.149

$\mathrm{Hu}, \mathrm{W} . \mathrm{F}$. (2000). Study of cultural differences in self-concept between Uygur and Han nationality students. Journal of Qinghai Nationalities Institute (Social Sciences), 26, 117-122.

Li, C. Z., Gan, X., \& Han, R. S. (2010). Relationship between self-concept and subjective well-being among senior high-school students. Journal of Liaoning Medical University (Social Science Edition), 8, 65-67.

Li, Z. S. (2006). Effects of undergraduates' cultural orientation and self-concept on their subjective well-being. Journal of Psychological Science (China), 29, 423-426.

Lin, B. J. (1980). The revision of Tennessee self-concept scale. Journal of China Testing Annual (Taiwan China), 27, 71-78.

Ling, Y., Zhu, C. Y., \& Liu, W. L. (2008). The correlative study on college students' subjective well-being and self-concept. China Journal of Health Psychology, 16, 1351-1352

$\mathrm{Mu}, \mathrm{D}$. (2000). Cross-cultural study on development of self-concept of students in junior high school in Inner Mongolia. Journal of Inner Mongolia Normal University (Philosophy \& Social Science), 29, 94-99.

Qiu, P. (2006). The study on self-awareness of Yi nationality. Journal of Guangxi Normal University (Social Science Edition), 27, 10-12.

Qiu, X. F., Zhang, W., \& Yao, D. J. (2007). The relationships of university teachers' well-being and coping strategies. Journal of the Modern Education Journal, 133, 22-26.

Qiu, X. F. (2009). A correlative study on coping styles and subjective well-being for teachers in university. Journal of Heilongjiang Researches on Higher Education, 185, 131-133.

Ren, Z. H., \& Ye, Y. D. (2006). Review on domestic and overseas researches of affecting factors of subjective well-being. Journal of Fujian Normal University (Philosophy and Social Sciences Edition), 139, 152-157.

Rim, Y. (1993). Happiness and coping styles. Journal of Personality and Individual Difference, 14, 617-618. doi:10.1016/0191-8869(93)90158-Y

Terry, T., \& Huebner, E. S. (1995). The relationship between selfconcept and life satisfaction in children. Journal of Social Indicators 
Research, 35, 39-52. doi:10.1007/BF01079237

Tong, Y. H. (2004). A correlative study on general self-efficacy, coping styles and subjective well-being in college students. Journal of Chinese School Health, 24, 396-397.

Wang, F., \& Fan, X. H. (2009). The study of the relationship of rural children's social support, self-concept and coping styles. Journal of Theory and Practice of Contemporary Education (China), 1, 149153.

Wang, J. S., \& Ding, X. H. (2003). A study on the correlation of subjective well-being and coping styles for junior middle school students. China Public Health, 19, 1181-1182.

Wang, X. D., Wang, X. L., \& Ma, H. (1999). Handbook of mental health scale. Beijing: Mental Health Magazine.

Wang, Z. H. (2001). A correlative study on self-concept and coping styles of junior high school students, Psychological Development and Education (China), 17, 22-27.

Wang, X. Y. (2008). A study of SWB and self-concept of the college youth sports teachers. Journal of Shandong Sports Science \& Technology, 30, 69-71.

$\mathrm{Wu}, \mathrm{L}$. H. (2008). Relationship between subjective well-being and self-concept of the old people. Chinese Journal of Gerontology, 30, 1873-1874.

Wu, M. X. (2000). Theoretical development of subjective well-being in the West for 30 years. Journal of Developments in Psychology (China), 4, 23-28.

Xin, Z. Q., Guo, S. R., \& Chi, L. P. (2007). The relationship of adolescents' self-esteem and aggression: The role of mediator and moderator. Journal of Acta Psychological Sinica, 39, 845-851.

Yang, H. R., \& Shi, G. X. (2004). A study on the correlation of subjective well-being and mental health of junior school students. Chinese Journal of Health Psychology, 12, 416-419.
Yang, H. S. (2008). The comparison between Tibetan and peripheral nationalities. Journal of Southwest University for Nationalities (Humanities and Social Science), 204, 203-207.

Zhang, L., \& Xu, Q. (2007). Effects of undergraduates' self-concept, collective self-esteem and individual self-esteem on their subjective well-being. Chinese Journal of Clinical Psychology, 15, 609-611.

Zhang, X. G., He, L. G., \& Zheng, X. (2004). The construct and scale formation of adolescent students' life satisfaction. Journal of Psychological Science, 27, 1257-1260.

Zhang, Y. H., \& Zhao, Z. R. (2007). The study of self of primary school students and middle school students of Han, Uighur and Kazak nationalities in Xinjiang. Journal of Tribune of Social Sciences in Xinjiang, 95, 72-75.

Zhao, X. D., \& Luo, T. (2010). Interaction between model writing with words and cultural integration-The social history of the development of the Qiang language and the identification of the Qiang minority. Journal of Guangxi University for Nationalities (Philosophy and Social Science Edition), 32, 78-84.

Zheng, K. M., Hao, Z. H., Hou, H. et al. (2008). A correlative research on coping style and self-concept of higher professional education students. China Journal of Health Psychology, 16, 877.

Zhou, A. B., Gao, X. D., \& Liu, Y. H. (2005). A comparative study on characteristics of sense of self-value of ethnic university and college students. Journal of Research on Education for Ethnic Minorities, 16, 58-65.

Zhou, T. M. (2009). Predictive effects of Han and Tibetan middle students' coping strategies in the face of mockery and loneliness, Journal of Neijiang Normal University, 24, 86-90.

Zhu, X. H., Miao, Y. J., \& Chen, H. B. (2008). Research progress on subjective well-being in the realm of social psychology abroad. Journal of Science of Social Psychology, 23, 23-27. 